\title{
Lucia Omacini (a cura di), L'opera incompiuta
}

\section{Andrea Schellino}

\section{(2) OpenEdition}

\section{Journals}

\section{Edizione digitale}

URL: http://journals.openedition.org/studifrancesi/6482

DOI: 10.4000/studifrancesi.6482

ISSN: 2427-5856

\section{Editore}

Rosenberg \& Sellier

\section{Edizione cartacea}

Data di pubblicazione: 1 novembre 2010

Paginazione: 605-607

ISSN: 0039-2944

\section{Notizia bibliografica digitale}

Andrea Schellino, «Lucia Omacini (a cura di), L'opera incompiuta», Studi Francesi [Online], 162 (LIV | III) | 2010, online dal 30 novembre 2015, consultato il 09 janvier 2021. URL: http://

journals.openedition.org/studifrancesi/6482 ; DOI: https://doi.org/10.4000/studifrancesi.6482

\section{Questo documento è stato generato automaticamente il 9 janvier 2021}

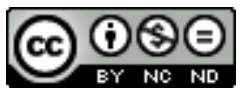

Studi Francesi è distribuita con Licenza Creative Commons Attribuzione - Non commerciale - Non opere derivate 4.0 Internazionale. 


\title{
Lucia Omacini (a cura di), L'opera incompiuta
}

\author{
Andrea Schellino
}

\section{NOTIZIA}

LUCIA OMACINI (a cura di), L'opera incompiuta, Annali di Ca' Foscari. Rivista della Facoltà di Lingue e Letterature Straniere dell'Università Ca' Foscari di Venezia, XLVII, 2, Studio Editoriale Gordini, 2008, pp. 256.

1 Il volume costituisce il prolungamento di una riflessione di ampio respiro sulla discontinuità letteraria inaugurata dal convegno sulla scrittura frammentaria (Théorie et pratique du fragment) tenutosi nel 2002 nell'ateneo veneziano. Come spiega Lucia OMACINI nell' Introduzione, la transizione dalla frammentazione all'incompiuto è relativamente breve e trova un propizio terreno d'indagine nell'opera spezzata e ferita del tournant des Lumières, in cui il vulnus della dilazione, del residuo e dell'incompiutezza mortifica e stravolge l'attesa dei lettori e dei teorici. La destrutturazione del modello classico non impedisce peraltro che la ricerca formale e l'idée de rapport, suggerita dai Salons di Diderot, restino una preoccupazione - un'àncora, una strategia - fondamentale dell'atto letterario; da questo punto di vista si fanno strada perciò l'idea di bello irregolare ed impuro, di cui il Romanticismo farà tesoro, e una garanzia di compiutezza e ordine che prescinde dal piatto sviluppo della fabula. Una chiusura può quindi prefigurare un incompiuto, mentre nulla osta che il significato di un'opera possa passare per le contraddizioni, gli strappi e persino i vuoti della narrazione - così Claude Simon: «Je ne comble pas les vides, ils demeurent comme autant de fragments» (p. 14). Per questa ragione unità e senso che vanno al di là della linearità del racconto sembrano esigere una vera ermeneutica della ricezione: finalmente la lettura, in quanto comprensione e interpretazione, si costituisce come polo forte e attivo della pratica letteraria (pensiamo al ruolo del lettore di fronte all'instabilità e alla "performatività" del Divertissement forain e dell'Ambigu-comique, o davanti alle suites inattese e al feuilleton, destinato a procrastinare o eludere il finale). 
L'ambizione di questa miscellanea, come è ben messo in luce da Lucia Omacini, non è solo quella di presentare alcuni casi emblematici di incompiutezza nella letteratura occidentale moderna, ma anche di accostarli ad esperienze del non-finito culturalmente differenti, da cui spiccano pertanto maniere stilistiche e orizzonti di scintillante originalità e ricchezza; la scommessa di uno sguardo su un panorama così esteso è sorretta da una scrupolosa sensibilità storica, che sottende ad ogni articolo.

2 La prima sezione del libro, dal titolo «Forme dell'incompiuto», si apre con lo studio di Jean-Paul SERMAIN (La poétique de l'inachèvement mise en cuvre par le roman aux XVII et XVIII siècles, pp. 19-38), in cui l'A. intende mostrare gli slittamenti e le sfumature di senso che l'incompiutezza dei romanzi acquista in epoche e contesti diversi. Nei racconti picareschi e nei romanzi epistolari ad esempio l'incompletezza non rappresenta un limite o un disturbo per il lettore. Al centro dell'articolo vi è però la tesi per cui la fine dell'intrigo, l'ultima avventura dell'eroe romanzesco, non va confusa-spesso al contrario se ne affranca e si contrappone ad essa - con il significato dell'opera, ossia ciò che emerge dal resto del romanzo; in questo senso sono analizzati Don Quijote de la Mancha, Madame Bovary, Les Liaisons dangereuses e La Nouvelle Héloïse. Loretta INNOCENTI ("Desunt nonnulla». La tragedia incompiuta in "Hero and Leander" di Christopher Marlowe, pp. 39-61) ricostruisce, sulla scia di Marion Campbell, la questione interpretativa che pone il poemetto di Marlowe Hero and Leander, a lungo considerato un'opera incompiuta. Per l'A. la cifra della composizione si trova «non nel tendere verso un fine, bensì nel senso di unione delle parti, della conciliazione degli opposti» (p. 53); nel testo convergono dunque due prospettive: quella tragica del mito, che conduce a pensare ad un'opera incompiuta, e quella comica e divertita del narratore, che allude alla completezza semantica e formale del testo. Questo poemetto bifronte non abbisogna per l'A. della fine luttuosa del mito, poiché nelle sue righe sono già rappresentate la sintesi degli opposti e la circolarità incantata che rendono «ripetibile quell'atto di amore nella poesia» (p. 58). Decentrato rispetto a queste questioni è l'articolo di Gian Giuseppe FILIPPI (L'incompiuto di Mamallapuram, pp. 63-81), dedicato alla parete scultorea incompiuta di Mamallapuram; l'A., attraverso una serrata descrizione dei miti indiani e dei bassorilievi, dimostra che essa raffigura una complessa narrazione su pietra della gangvatrana, la discesa del Gange sulla terra. Questa composizione cosmografica è la medesima dell'opera compiuta di Mamallapuram, evidentemente iniziata dopo che una folgore, presagio di sventure, ebbe danneggiato il primo monumento.

3 Liminare alla seconda sezione del volume, "L'estetica del frammento», è l'articolato studio di Bettina FABER ( «Und vieles wie auf den Schultern eine Last von Scheitern ist zu behalten». Moderner Fragmentarismus und Hölderlins anderes Bewusstsein von der Bedeutung des Unvollendeten, pp. 85-130), che indaga alcuni spazi privilegiati del rapporto tra frammento e totalità, tra finitezza e nostalgia del divino. L'A. conduce il suo percorso dall'interesse post-moderno per il frammento à rebours fino alle aporie del movimento fondativo del primo Romanticismo tedesco. La fragilità dell'umano e la sua radicale incompletezza si rivelano appieno nella poesia di Hölderlin, drammatica constatazione della rottura dell'uomo con l'infinita totalità della natura; da un'attenta lettura di Hyperion, del frammento su Empedocle, dei grandi Inni, emerge la «Dramatizität der existentiellen Situation des Menschen und Dichters» (p. 127), ma soprattutto l'odissea irta di vuoti, scacchi ed errori del poeta moderno che mantiene un contatto con gli dèi e tenta di farne partecipe l'umanità. Eloisa PAGANELLI (Frammentazione scenica e discorsiva nel teatro di John Webster: una modalità dell'incompiuto nell'ambito della crisi giacomiana, 
pp. 131-154) esamina i due capolavori teatrali di Webster, The White Devil e The Duchess of Malfi, per mostrare come la tecnica compositiva del misterioso scrittore inglese (la messa in scena delle potenze ctonie e disgregatrici e della progressiva dissociazione psicologica dei personaggi, la frantumazione del linguaggio e della versificazione, l'ellissi e l'opacità) trovi corrispondenza nella crisi coeva della rappresentazione circolare; ad essa subentra la linea serpentina e spiralata, gravata d'inquietudini ed incertezze (M. Nicolson). Paola MARTINUZZI (Dal divertissement forain all'ambigu-comique: l'arte di frantumare e di combinare, pp. 155-165) descrive la tendenza dei generi popolari teatrali tra la fine del xvII e l'inizio del xviII secolo a sottrarsi alla definizione e all'unicità, preferendo ad esse la ricreazione a contatto con il pubblico; questa mobilità combinatoria, potenzialmente aperta ed aleatoria, è un tipico caso di incompiutezza programmatica. L'articolo di Nicoletta PESARO ( $\mathrm{Su}$ Manshu: la modernità incompiuta o l'incompiutezza del moderno?, pp.167-188) presenta alcuni aspetti della tormentata personalità e della scrittura di Su Manshu, autore a cavallo tra cultura cinese e giapponese, tra tradizione e modernità, tra Oriente ed Occidente. L'analisi dell'impianto narratologico della sua opera consente di apprezzare l'indefinitezza, nonché la quête stessa di una trama («intermittenza strutturale del cuore», p.178), elementi che costituiscono il fascino della sua narrazione.

4 La terza e ultima parte del volume, «Il nulla e lo zero», mostra al contempo il lembo estremo e periglioso della frammentazione, le occasioni che esso dona e le rive più lontane dalla tradizione occidentale. Il contributo di Silvia BURINI (Il fecondissimo nulla: alcuni esempi di semiotica dello zero nel concettualismo russo, pp. 191-223) analizza i tratti peculiari del concettualismo moscovita tramite la considerazione di alcune produzioni ibride tra arti figurative e letteratura. Di fronte all'onnipervasività dell'ideologia ufficiale sovietica l'unica forma artistica diviene l'assenza della forma, il nulla ed il vuoto in tutte le sue ipostasi; gli Album di Kabakov denunciano la realtà ideologizzata $\mathrm{e}$ al termine del viaggio il personaggio è atteso dal bianco, dal nulla. Lo stile dell'assenza (Roland Barthes), il punto zero, è sorto secondo Epštejn dalla ridondanza e dall'eccesso novecenteschi-l'iper - che hanno tradito, per antitesi, la loro completa vacuità e mancanza di senso; ciononostante Malevich delinea nella sua arte un Nulla che sprigiona un'enorme forza creativa, un «seme dell'infinito» (p. 212). Un altro sbocco possibile è per l'A. paradossalmente offerto dall'estetica della spazzatura e dei rifiuti, che svela il volto tragico della merce fino a costituirsi come museo-cassonetto e nuovo luogo della memoria. Conclude il volume lo studio di Ornella CIVARDI (Quello che manca al Nulla. Forme dell'incompiuto e tecniche di salvazione nella cultura giapponese, pp. 225-243) la quale contrappone alla concezione occidentale del frammento e dell'imperfezione consunta dal rimpianto della totalità e della purezza perdute - la serena attitudine dell'incompiutezza nipponica: «più riflesso che frammento, non rimanda a un Tutto altro da sé, ma a una totalità che risiede al suo interno, seme sempre pronto a germogliare» (p. 226). In Giappone l'impuro e l'abbozzato sono ammirabili poiché vi risplendono la schietta spontaneità della natura e la scintilla vitale del divino. 